\title{
Study of crystal size evolution by focused-beam reflectance measurement during the freezing of sucrose/water solutions in a scraped-surface heat exchanger
}

\author{
Anis Haddad Amamou ${ }^{\mathrm{b}, \mathrm{c}}$, Hayat Benkhelifa ${ }^{\mathrm{a}, \mathrm{c}, *}$, Graciela Alvarez $^{\mathrm{b}, \mathrm{c}}$, Denis Flick ${ }^{\mathrm{a}, \mathrm{c}}$ \\ a UMR 1145 Génie Industriel Alimentaire, AgroParisTech, 16 rue Claude Bernard, 75231 Paris Cedex 05, France \\ b UMR 1145 Génie Industriel Alimentaire, Cemagref Unité GPAN, Parc de Tourvoie BP 44, 92163 Antony, France \\ c UMR 1145 Génie Industriel Alimentaire, INRA, France
}

This study examines the freezing step that occurs in a scraped-surface heat exchanger during the manufacturing of sorbet. During this step, the product enters the exchanger as a liquid, then it is cooled and partially crystallized before exiting the exchanger as a mixture of liquid and ice, also called sorbet. The freezing step governs the final quality of the product, particularly its texture. Most existing studies have focused on the product after freezing at the exchanger exit. The aim of this work was both to follow the evolution of the ice crystals' granulometry during the freezing of sorbet in the exchanger and to relate this evolution to process parameters such as refrigeration temperature, scraper speed and initial sucrose concentration. Few in situ sensors exist to follow granulometry, and this fact is especially true for rapid kinetics and concentrated suspensions. Focused-beam reflectance measurement (FBRM), an original tool, was used in this study. FBRM is currently used in the chemical and pharmaceutical industries to follow product granulometry, but it is not used in food-related applications. In our study, an experimental protocol was developed to assure identical initial thermal and crystallization conditions. First, the sensor sensitivity and the repeatability of the results were verified. The measurements performed with the FBRM probe showed that this technique can be used to follow crystal granulometry in a sorbet consisting of up to $30 \%$ of ice. The effect of process parameters was then analyzed. It appears that a decrease in refrigeration temperature accelerates ice crystallization and yields slightly smaller crystals. The same effect is observed with increasing scraper speed. Additionally, when the initial sucrose concentration in the solution is increased, the ice fraction increases more slowly but the mean chord length is smaller.

\author{
Keywords: \\ Freezing \\ Sucrose solution \\ Ice crystallization \\ Crystal size evolution \\ Scraped-surface heat exchanger \\ Focus beam reflectance measurement
}

\section{Introduction}

The smooth texture and creamy mouthfeel of sorbet and ice cream are two sensory characteristics of these products that are most appreciated by consumers. A small mean ice crystal size $(10-20 \mu \mathrm{m})$ is essential to give the product its desired texture [1]. Ice crystals larger than about $50 \mu \mathrm{m}$, if present in sufficient number, confer a coarse or grainy texture to the final product [2]. To control ice crystal size distribution, it is important to understand how crystals both are formed and grow during the manufacturing process. Formation of ice occurs within two stages of the ice cream manufacturing process: after the initial freezing in a continuous scraped-surface heat exchanger SSHE (known as the freezer) and then during the hardening in a blast freezer where air (at around

\footnotetext{
* Corresponding author at: UMR 1145 Génie Industriel Alimentaire, AgroParisTech, 16 rue Claude Bernard, 75231 Paris Cedex 05, France. Tel.: +33 01409662 27; fax: +330140966075.

E-mail address: benkheli@agroparistech.fr (H. Benkhelifa).
}

$-25^{\circ} \mathrm{C}$ ) is the cooling medium. During hardening, undercooling is not sufficient to allow the nucleation of new crystals, but ice phase volume increases due to the growth of existing nuclei [3]. Because of this observation, the properties of the crystal population in the hardened product are largely determined by the initial freezing. Therefore, it is important to better understand the ice crystallization mechanisms and the proceeding parameters that affect them.

In a conventional ice-cream freezer, the first ice crystals appear at the barrel wall at a rate determined by the local subcooling [4]. This subcooling is produced by the use of an evaporating refrigerant such as ammonia. Ice crystals are then removed from the barrel wall by scraper blades that are attached to a rotating shaft. The scrapers are slightly warmer due to the release of latent heat and mechanical energy into the system. Schwartzberg and Liu [5] proposed the following mechanism for ice formation in a scraped-surface freezer. Dendritic crystals are formed on the scraped-surface wall, grow and are scraped off the wall and dispersed into the bulk. They then ripen into the block-shaped crystals seen upon exit from the freezer. The process of such crystallization is complex and involves many coupled interactions between fluid flow, heat transfer and ice crystal 


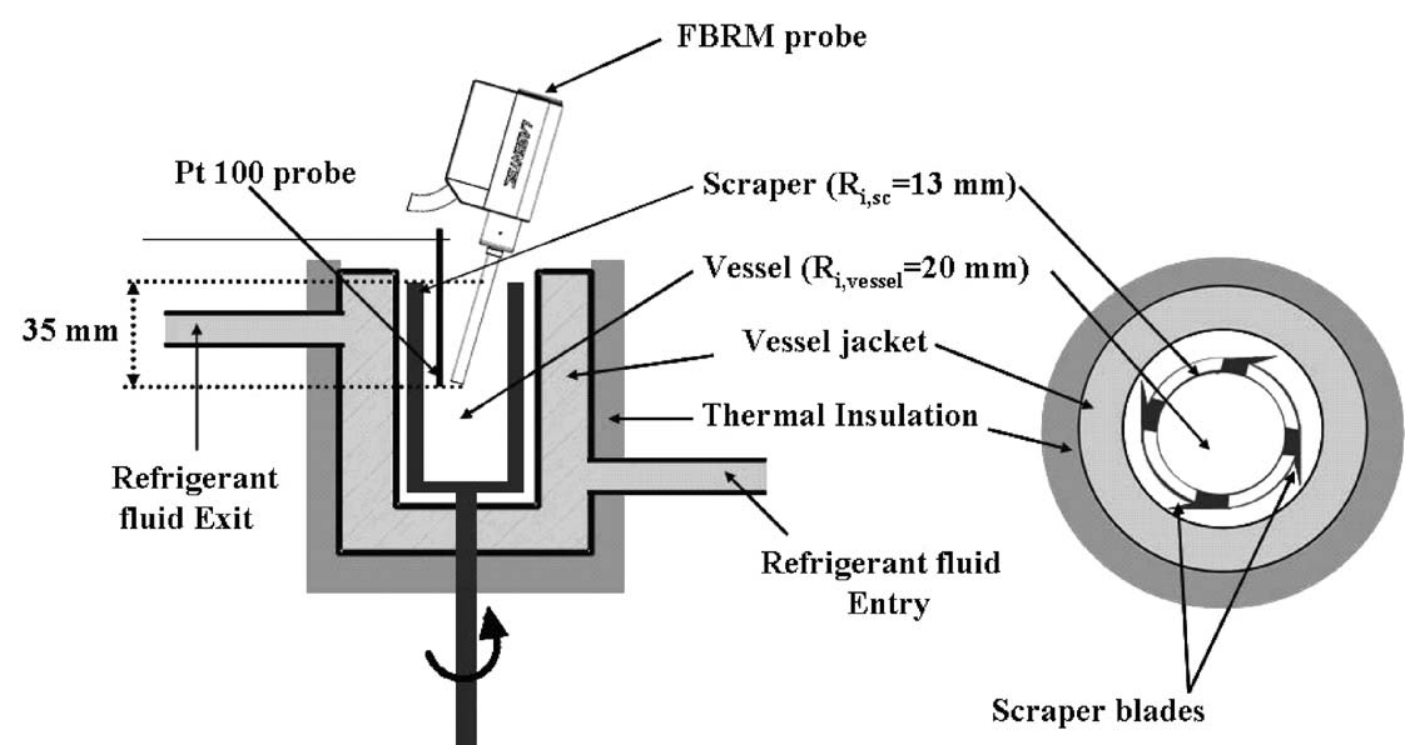

Fig. 1. Experimental device: experimental batch SSHE and positions of FBRM and Pt100 probes in the vessel of exchanger.

nucleation and growth [6]. The kinetics of ice crystallization in the SSHE and the influence of process conditions on the crystal size distribution (CSD) are not very well elucidated. Control of the ice crystallization process is still usually empirical, essentially because of a lack of experimental data. In fact, ice CSD measurement is not simple because of both the possibility for melting the ice and the complex nature of an ice cream formulation, which contains three phases. In-line and real-time measurement of CSD were recently developed to prevent the perturbations in temperature, cooling rate and supersaturation observed during sampling or when using external recirculation in off-line techniques.

One of these developed techniques is the focused-beam reflectance measurement (FBRM). This instrument is increasingly used to investigate and monitor CSD during industrial crystallization processes. This tool offers a solution to the classic light scattering techniques that provide useful CSD measurements but require a sampling and dilution unit. The FBRM technique overcomes these limitations by focusing a laser beam through a window in the probe tip and then collecting the laser light that is scattered back to the probe [7]. In fact, the FBRM probe emits a laser beam rotating at a high velocity $(9000 \mathrm{rpm})$, which propagates into the suspension through a sapphire window. Particle motion is therefore insignificant to the measurement [8]. The beam intersects particles suspended in the solution and is backscattered until it reaches the opposite edge of the particle. This back-scattered light is collected by the FBRM optics and is converted into chord length as the product of the measured crossing time and the beam velocity [9]. The instrument can acquire thousands of chord lengths per second. On a counter board, these lengths are classified into a series of size ranges and are expressed as a distribution, which is referred to as the chord length distribution (CLD). The most attractive advantage of this technique is its utility both for in situ measurement of high solid-concentration suspensions and for following rapid crystallization kinetics. However, the CLD is only an indication of the real population. This probe does not detect particle shape. In fact, CSD is more meaningful for the characterization of a crystal product. Thus, many papers have discussed methods to recover the CSD value from a measured CLD. If the crystal shape is known, particularly when the crystals can be considered as spherical, this restoration is possible. Furthermore, if crystal shape does not change during freezing, the mean chord length can be considered proportional to the mean diameter.
The FBRM technique has been used widely in both continuous [10] and batch crystallization [11,12]. However, there are no published studies regarding the use of this technique during ice crystallization in SSHE. Furthermore, the studied materials were mainly chemical and pharmaceutical products, but there have been no previously reported studies on ice crystallization.

The purpose of this work was to use the FBRM technique to study ice crystal evolution during the freezing of sugar solutions in a batch SSHE. The sensor sensitivity and the repeatability of the results were studied in order to determine the probe's performance. Several processing and formulation parameters were then studied and related to crystallization kinetics, especially to the ice crystal size, with a view to understanding the dominant crystallization mechanisms within an SSHE.

The batch SSHE used in this study is a laboratory scale batch exchanger. In this exchanger, the scraper blades are located $1 \mathrm{~mm}$ from the cooling surface, while in an industrial freezer the scraper blades are generally in contact with the cold inner surface. In the present case, a 1-mm ice layer is probably formed on the cold wall, which increases the internal heat transfer resistance. Previous studies were performed in our laboratory [13] in a pilot scraped-surface heat exchanger, and they were concerned with the effect of the distance between the blades and the cold wall. Temperature measurements at the wall were performed, and the inner heat transfer resistance was determined for distances between the blades and the cold surface of $1-3 \mathrm{~mm}$. It was shown that a permanent ice layer forms only when the gap between the blades and the wall is large enough (for a distance of $3 \mathrm{~mm}$ ), otherwise the ice layer is not strong enough and is periodically removed from the wall (for $1 \mathrm{~mm}$ ). In the present study, we considered that, even if a 1-mm ice layer was formed on the wall, this layer was not permanent. Therefore, the conditions that were studied can be considered relatively close to those of an industrial freezer.

\section{Materials and methods}

A batch scraped-surface heat exchanger was designed (Fig. 1) and constructed by the Refrigeration Process Engineering Research Unit at the Cemagref. It consisted of a cylindrical cup of $100 \mathrm{ml}$ (height, $120 \mathrm{~mm}$ and inner diameter, $40 \mathrm{~mm}$ ) equipped with a vessel jacket, where the circulation of a refrigerant fluid allows the cooling of the product. The scraper, driven by a variable speed motor and controlled by a non-contact type tachometer, consisted of four stainless steel blades located $1 \mathrm{~mm}$ from the wall. The blades were set on a hollow cylindrical support with an internal radius $R_{\mathrm{i}-\mathrm{sc}}$ of $13 \mathrm{~mm}$. 


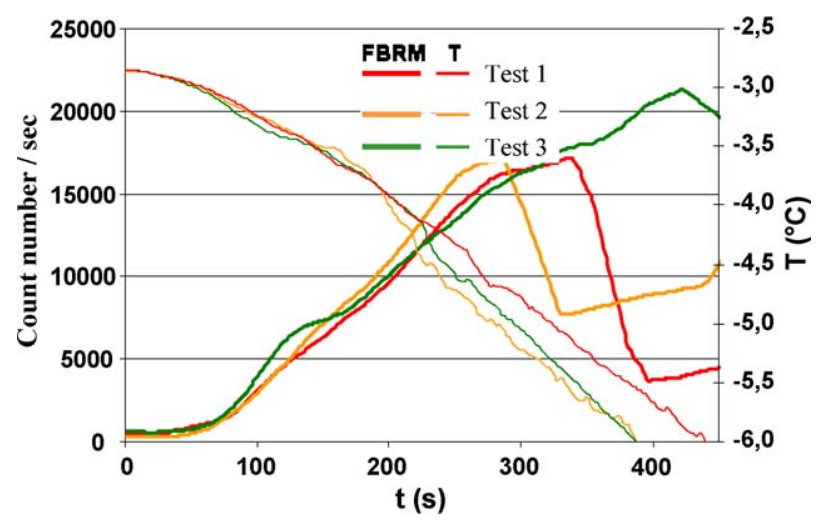

Fig. 2. Temperature and chords count number per second as a function of time for three replicates in the reference conditions.

As shown in Fig. 1, the FBRM probe S400 (Lasentec, Mettler Toledo) and the Pt100 probes were immersed in the SSHE vessel containing the solution to be studied. The FBRM probe was equipped with a nitrogen purge system to prevent condensation on the sapphire window when the temperature fell below $5{ }^{\circ} \mathrm{C}$. The probe allowed for the determination of the total number of counts per second and counts per class (CLD) as well as the mean chord length during the freezing step. The Pt100 sensor (1.6 mm diameter) measured the product temperature with an accuracy of $\pm 0.03^{\circ} \mathrm{C}$. From this temperature measurement, the ice mass fraction $\left(X_{\mathrm{mg}, \mathrm{eq}}\right)$ was determined by assuming thermodynamic equilibrium and temperature uniformity in the slurry. The equilibrium curve of the water/sucrose mixture obtained from the relationship proposed by Bubnik et al. [14] was used to determine the sucrose concentration in the residual solution and the ice mass fraction as a function of the slurry temperature.

The freezing of the studied solutions was carried out in two stages. An experimental protocol was developed in order to assure the same initial thermal and crystallization conditions for all the tests. Seeded crystals were previously prepared in the same exchanger and stored at $-18^{\circ} \mathrm{C}$. During the first stage, the solution was stabilized at a defined temperature below its freezing point and a small controlled amount of ice crystals was seeded in the vessel to initiate crystal nucleation. The supercooling phenomenon, which can randomly happen, is thus avoided. At the end of this stage, the ice content for all the studied solutions was nearly $3 \%$. This point is the initial freezing state.

In the second stage, the examined freezing step was started using a lowtemperature refrigeration bath. The reference conditions were: a bath refrigerating fluid temperature of $-20^{\circ} \mathrm{C}$, a scraper speed of $300 \mathrm{rpm}$ and a solution containing water and $30 \%$ sucrose.

In this work, the effects of three parameters were studied:

- The bath refrigerating fluid temperature was set at $-10,-20$ and $-30^{\circ} \mathrm{C}$, with a scraper speed equal to $300 \mathrm{rpm}$ and a solution with an initial sugar content of $30 \%$. - The scraper speed was set at 25,50,100, 300 and $400 \mathrm{rpm}$, with a refrigeration temperature of $-20^{\circ} \mathrm{C}$ and a solution with an initial sugar content of $30 \%$.

- The initial sugar content of the solution was set at 15,30 and $45 \%$, with a refrigeration temperature of $-20^{\circ} \mathrm{C}$ and a scraper speed equal to $300 \mathrm{rpm}$.

\section{Results}

\subsection{Probe sensitivity and result repeatability under the reference conditions}

After performing three tests using the same reference conditions, Fig. 2 shows the evolution of the total count number (number of particles detected per second) and of the sorbet temperature near the cup center as a function of time. The solution was initially at $-2.85^{\circ} \mathrm{C}$ and contained nearly $3.5 \%$ (mass fraction) ice. The vessel jacket was fed with the refrigerating fluid at $-20^{\circ} \mathrm{C}$. After nearly $10 \mathrm{~s}$, a time that corresponds to the thermal resistance of the solution, the solution temperature decreased. The count number per second then increased, which corresponds to the formation of new ice crystals.

However, it should be noted that the increase in this count number begins with a greater time delay (around $40 \mathrm{~s}$ ) than the thermal inertia observed. This observation can be explained by the fact that the solution is refrigerated at the cold wall and arrives quickly at the center by radial mixing, but the solution does not initially contain

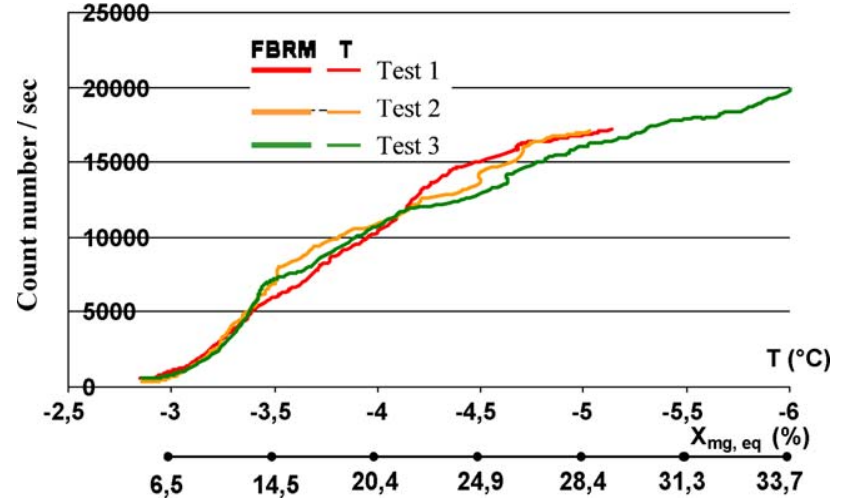

Fig. 3. Chords count number per second as a function of temperature for three replicates in the reference conditions.

new crystals. Time is required for nucleation to begin at the wall, and the $1-\mathrm{mm}$ ice layer has to be scraped. Unfortunately, the FBRM probe cannot detect crystal shape changes, and several shapes can give the same chord distribution. However, the main goal of the use of this probe is that the size changes are detected and can be quantified.

Next, the total count number increases regularly with solution cooling and finally falls sharply after nearly 300 s. This fall can be explained by the significant amount of ice (around 30\%) in the sorbet, which begins to stick to the probe and disturb the measurement. It is important to recall that the FBRM measurement can be dependent on stirring performance and crystal density, particularly near the probe (Heath et al. [15]). The stirring must be sufficient to avoid ice sticking.

At around $200 \mathrm{~s}$, the thermal evolution of the three tests becomes significantly different. Visual observation shows that the higher the amount of ice, the more the flow is disrupted. This disruption influences the heat transfer and the radial mixing and therefore the temperature measurement. To overcome this variability, it is better to represent the count number evolution as a function of temperature (and equivalent ice mass fraction) instead of time (Fig. 3). In this figure, the three curves are close to one another, meaning that the evolution of the count number is similar for the three replicates. The measurements performed with the FBRM probe can then be considered correct and repeatable for an amount of ice up to $30 \%$.

\subsection{Refrigeration temperature effect}

First, the results are presented in terms of the evolution of mean chord length as a function of temperature or ice mass fraction (Fig. 4). Each curve is an average of three tests and the error bars are included. Second, the count number percentage as a function of chord length, which corresponds to the CLD at a given temperature, is presented (Fig. 5). It was observed that for the three refrigeration temperatures tested, the mean chord length decreased rapidly at first until the ice content reaches nearly $15 \%$. This change can be explained by the fact that when freezing first begins, the nucleation of new small crystals (smaller than the seeded crystals) is the predominant effect leading to the decrease in the mean chord length. This decrease occurs even faster when the refrigerating temperature is low (especially when $\mathrm{Tb}=-30^{\circ} \mathrm{C}$ ) because the faster new crystals are created, the faster the relative importance of the large crystals decreases.

Next, crystal growth occurs, but seems to be limited by the attrition of large crystals at high ice content or by melting. Above an ice content of $20 \%$, the ice crystal size seems to stabilize at a constant value. This phenomenon was observed in a similar study by Lian et 


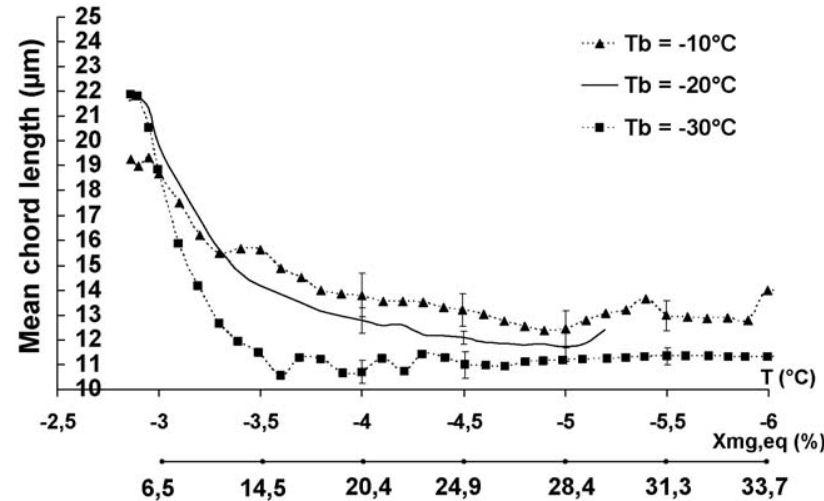

Fig. 4. Effect of refrigeration temperature on mean chord length evolution (average of three tests) with solution temperature and the corresponding ice fraction assuming thermodynamic equilibrium.

al. [6]. It can also be noted that the faster the freezing, the smaller the mean crystal size when the temperature is less than $-3.5^{\circ} \mathrm{C}$ (corresponding to an ice content above 15\%).

The observation of the CLD when the mass ice concentration reaches about 25\% confirms this trend (Fig. 5). Under these conditions, the CLD shifts towards smaller sizes as the refrigeration temperature decreases. The CLD curves are similar (approximately log-normal) and most of the chord lengths are between 3 and $30 \mu \mathrm{m}$, with a slightly narrower dispersion when the refrigeration temperature decreases. The sorbet is therefore more uniform in size and contains a larger quantity of smaller crystals at the low refrigerating temperature.

\subsection{Scraper speed effect}

Fig. 6 shows the effect of scraper speed on the mean chord length evolution from an average of three tests, while assuming thermodynamic equilibrium for the solution temperature and corresponding ice fraction.

The scraper speed increase has the same global effect as that observed when reducing the refrigerant fluid temperature, i.e. a decrease in mean chord length and a CLD shifted towards small sizes as shown when the ice concentration reaches $20.4 \%$ (Fig. 7). By increasing the scraper speed, more crystals migrate from the wall to the center of the vessel. These crystals can generate other smaller crystals by secondary nucleation and/or attrition. On the contrary, the dissipation of mechanical energy into heat increases, which can lead to the melting of small crystals and result in an increase in the average size of crystals at high speed scraping. This effect was observed in some cases in the literature. One of these

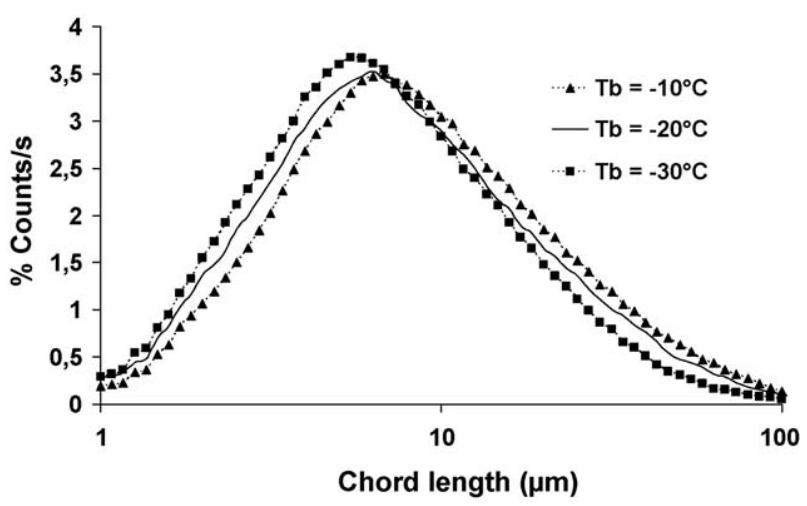

Fig. 5. Effect of refrigeration temperature on CLD at $-4.5^{\circ} \mathrm{C}$ (at ice fraction $X_{\mathrm{mg}, \mathrm{eq}}=24.9 \%$ assuming thermodynamic equilibrium).

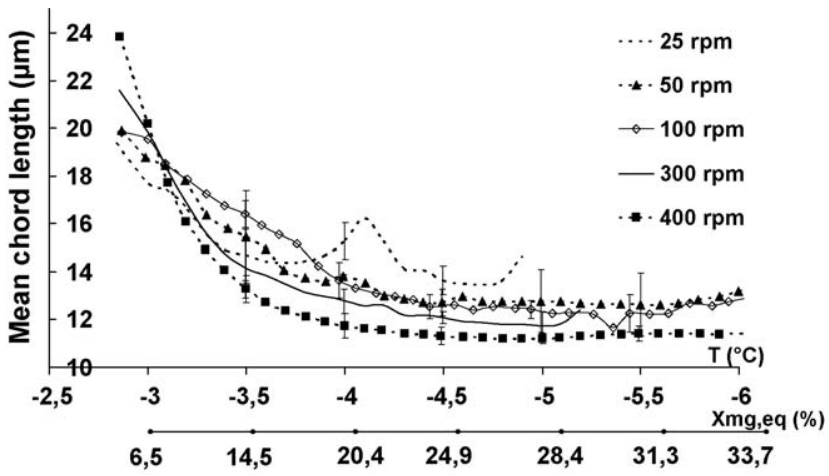

Fig. 6. Effect of scrapers speed on mean chord length evolution (average of three tests) with solution temperature and the corresponding ice fraction assuming thermodynamic equilibrium.

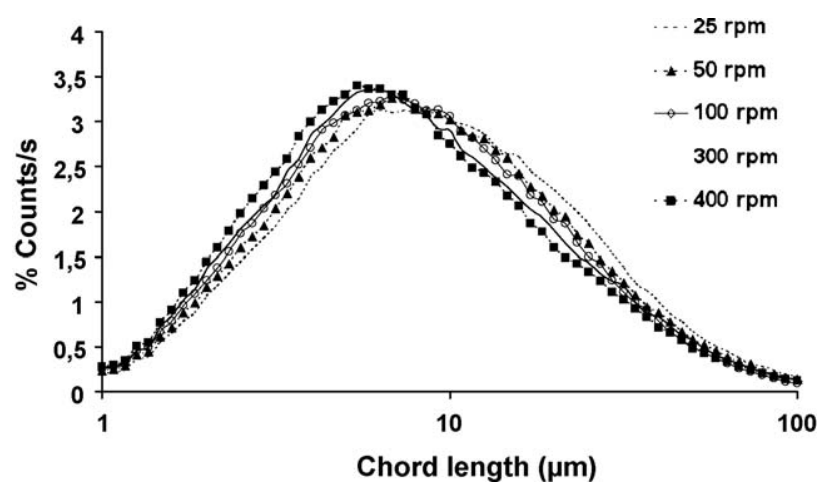

Fig. 7. Effect of scrapers speed on CLD at $-4{ }^{\circ} \mathrm{C}$ (at ice fraction $X_{\mathrm{mg}, \mathrm{eq}}=20.4 \%$ assuming thermodynamic equilibrium).

two opposite effects can be the predominant one. In our case, the results showed that the attrition effect is more important than heat dissipation.

\subsection{Sucrose concentration effect}

When increasing the initial sugar content, the ice fraction increases more slowly and the average crystal size decreases, as shown by the measured CLD at $X_{\mathrm{mg}, \mathrm{eq}}=25 \%$ (Fig. 8). Indeed, increasing the sucrose content of the solution leads to a higher viscosity. Therefore, there is more dissipation of mechanical energy into heat (possibly melting other crystals) and, more importantly, viscous strain (meaning higher attrition). On the other hand, the resistance

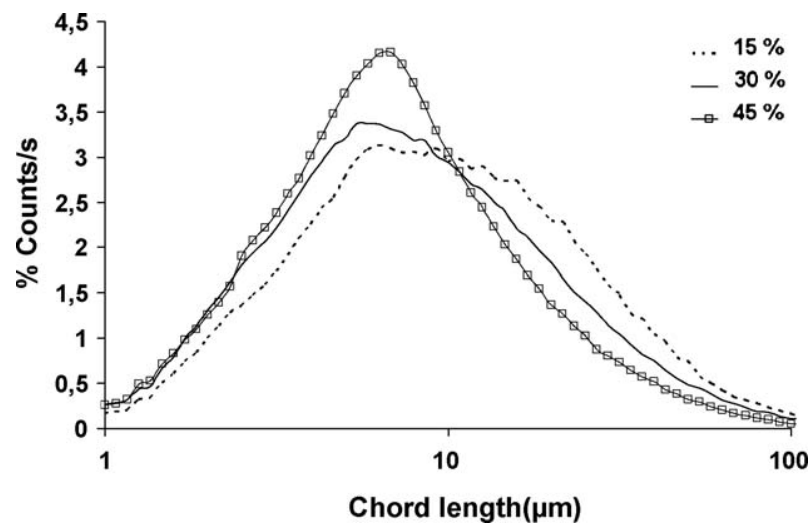

Fig. 8. Effect of sucrose concentration on CLD (at ice fraction $X_{\mathrm{mg}, \text { eq }}=25 \%$ assuming thermodynamic equilibrium) 
to water diffusion to the ice crystal surface is higher and the growth rate is, therefore, lower.

\section{Conclusions}

This study confirms that the FBRM technique can be considered a convenient tool for studying ice crystallization in terms of ice crystal size evolution during the freezing of sugar solutions in a batch SSHE.

The results seem to be repeatable, and the information given by the probe is similar to that obtained by other studies in the literature. The measurements seem to be reliable for an ice content below $30 \%$, which can be considered a promising result in comparison with other existing techniques.

The studied parameters show the expected effects in terms of the measured mean chord lengths and CLD. Indeed, a decrease in the refrigeration temperature leads to smaller crystals by increasing the undercooling and accelerating the ice crystallization rates. An increase in scraper speed shows the same effect. The decrease in average crystal size can be caused by the attrition effect, which seems to be predominant when the scraper speed is high. However, on an industrial scale, increases in the refrigeration rate and scraper speed are limited by energy consumption considerations. Indeed, a compromise between smaller crystal size and energy consumption has to be found.

With regard to the effect of the initial sucrose concentration in the solution, it appears that the average crystal size decreases with increased sucrose concentration. This effect can be explained by two facts. First, the higher viscosity promotes crystal melting and attrition. Second, the solution has a higher resistance to water diffusion to the ice crystal surface.

Finally, it may be interesting to restore the real CSD from the CLD in order to have a more realistic description of the ice crystal population in the SSHE. The use of a convenient photo or video microscopic technique and image analysis could be helpful both for achieving this restoration and for confirming the observed effects reported here. This study is now in progress.

\section{References}

[1] Russell AB, Cheney PE, Wantling SD. Influence of freezing conditions on ice crystallisation in ice cream. Journal of Food Engineering 1999;39:179-91.

[2] Miller-Livney T, Hartel RW. Ice recrystallization in ice cream: interactions between sweeteners and stabilizers. Journal of Dairy Science 1997;80:447-56

[3] Sutton R, Bracey J. The blast factor. Dairy Industries International 1996;61(2):31-3.

[4] Fennema OR, Powrie WD, Marth EH. Low-temperature preservation of foods and living matter. New York: Marcel Dekker; 1973.

[5] Schwartzberg HG, Liu Yc. Ice crystal growth on chilled scraped surfaces. In: Summer National Meeting, Paper No. 2g. American Institution of Chemical Engineers; 1990.

[6] Lian G, Moore S, Heeney L. Population balance and computational fluid dynamics modelling of ice crystallisation in a scraped surface freezer. Chemical Engineering Science 2006;61:7819-26.

[7] Hukkanen EJ, Braatz RD. Measurement of particle size distribution in suspension polymerization using in situ laser backscattering. Sensors and Actuators B: Chemical 2003;96(1-2):451-9.

[8] Abbas A, Nobbs D, Romagnoli JA. Investigation of on-line optical particle characterization in reaction and cooling crystallization systems. Current state of the art. Measurement Science and Technology 2002;13(3):349-56.

[9] Worlitschek J, Mazzotti M. Choice of the focal point position using Lasentec FBRM. Particle \& Particle Systems Characterization 2003;20:12-7.

[10] Kougoulos E, Jones AG, Jennings KH, Wood-Kaczmarc MW. Use of focused beam reflectance measurement (FBRM) and process video imaging (PVI) in a modified mixed suspension mixed product removal (MSMPR) cooling crystallizer. Journal of Crystal Growth 2005;273:529-34.

[11] Worlitschek J, Mazzotti M. Model-based optimization of particle size distribution in batch cooling crystallization of paracetamol. Crystal Growth and Design 2004;4(5):891-903.

[12] Barthe S, Rousseau RW. Utilization of focused beam reflectance measurement in the control of crystal size distribution in a batch cooled crystallizer. Chemical Engineering and Technology 2006;29(2):206-11.

[13] Ben Lakhdar M, Cerecero R, Alvarez G, Guilpart J, Denis F, Lallemand A. Heat transfer with freezing in a scraped surface heat exchanger. Applied Thermal Engineering 2005;29(1):45-60.

[14] Bubnik Z, Kadlec P, Urban D, Bruhns M. Sugar technologists manual. 8th edition Germany: Verlag Dr. A. Bartes; 1995.

[15] Heath AR, Fawell PD, Bahri A, Swift JD. Estimating average particle size by focused beam reflectance measurement (FBRM). Particle \& Particle Systems Characterization 2002;19:84-95. 